\title{
MODELAGEM MATEMÁTICA DA EXTRAÇÃO DE ÓLEOS BIOATIVOS DE MICROALGAS USANDO FLUIDO SUPERCRÍTICO
}

\author{
D. BESSA ${ }^{1}$, A. L. B. SOUZA ${ }^{1}$, R. B. DERNER ${ }^{2}$ e M. F. MENDES ${ }^{1}$. \\ ${ }^{1}$ Universidade Federal Rural do Rio de Janeiro, Departamento de Engenharia Química, \\ Laboratório de Termodinâmica Aplicada e Bicombustíveis. \\ ${ }^{2}$ Universidade Federal de Santa Catarina, Departamento de Aquicultura, Laboratório de Cultivos \\ de Algas. \\ E-mail para contato: marisamf@ufrrj.br
}

\begin{abstract}
RESUMO - A utilização de fluidos supercríticos na extração de produtos naturais vem sendo utilizada na obtenção de compostos bioativos a partir da biomassa microalgal, que se destaca por apresentar em sua composição, proteína, ésteres, carotenoides, clorofila, enzimas, antibióticos, hidrocarbonetos e vitaminas. Diante disso, o presente trabalho tem como objetivo avaliar tecnicamente o processo de extração usando fluido supercrítico, empregando-se dióxido de carbono como solvente e modelar matematicamente o processo de extração do óleo da microalga Desmodemus sp. usando o modelo de Esquível et al. (1999). A unidade experimental consiste, basicamente, de um extrator de $42 \mathrm{~mL}$, uma bomba de alta pressão e uma válvula micrométrica para a amostragem do extrato. Foi utilizado um método convencional a fim de comparar com a extração supercrítica. Os melhores rendimentos em óleo foram nas condições operacionais de 300 bar e $100^{\circ} \mathrm{C}, 500$ bar e $70^{\circ} \mathrm{C}$ e 440 bar e $90^{\circ} \mathrm{C}$, alcançando valores de $3,342 \%, 3,103 \%$ e $2,586 \%$, respectivamente. O modelo de Esquível se mostrou apto para modelar as curvas experimentais da extração do óleo da microalga Desmodesmus sp.
\end{abstract}

\section{INTRODUÇÃO}

As microalgas são micro-organismos fotossintéticos que são conhecidos como os principais produtores primários dos ecossistemas aquáticos (Brennan \& Owende, 2010). Como são um grupo, elas podem lidar com altas e baixas temperaturas, intensidades de luz abaixo do ideal, baixa disponibilidade de nutrientes essenciais e outros recursos. Uma variedade de estratégias fisiológicas e bioquímicas proporciona às microalgas uma riqueza de moléculas orgânicas complexas que pertencem à categoria de produtos químicos bioativos, dependendo das vias biossintéticas (Skulberg, 2004).

A extração com $\mathrm{CO}_{2}$ supercrítico de moléculas de interesse a partir de microalgas atualmente se tornou um assunto de grande interesse e numerosos dados experimentais já estão disponíveis na literatura, entretanto, pouca informação é fornecida acerca dos modelos matemáticos utilizados nas curvas de extração. 
Os principais campos de aplicação é o setor da saúde com foco para alimentos e farmacêuticos para a extração de lipídeos ou moléculas dedicados ao consumo humano ou a saúde animal (Montero et al., 2005).

Diante do exposto, o objetivo deste trabalho foi avaliar tecnicamente o processo de extração usando fluido supercrítico e modelar matematicamente o processo, usando os modelo de Esquível et al. (1999), de modo a otimizar as condições de temperatura e pressão.

\section{MATERIAIS E MÉTODOS}

\subsection{Materiais}

A microalga utilizada nesta pesquisa, Desmodesmus sp., foi obtida e cultivada pelo Departamento de Aquicultura (CCA) da UFSC. A biomassa foi centrifugada, seca em estufa e mantida sob refrigeração e ao abrigo da luz até sua utilização. A microalga utilizada nesse trabalho é uma microalga dulcícola, com uma parede celular rígida e de dimensões típicas de cerca de 6-8 $\mu \mathrm{m}$ comprimento e $3 \mu \mathrm{m}$ de largura (Garcia-Alba et al., 2012).

Os solventes utilizados para os experimentos foram hexano P.A. (misturas de isômeros, Isofar, Rio de Janeiro, Brasil) e dióxido de carbono (mínimo de pureza de 99,998\%, White Martins, Rio de Janeiro, Brasil).

\subsection{Metodologia Experimental}

\section{Extração com Soxhlet}

Cerca de 5 gramas de biomassa microalgal foram pesadas e colocadas em um cartucho de celulose e alimentados em um extrator. Em um balão de $500 \mathrm{~mL}$ foram colocados $100 \mathrm{~mL}$ de solvente, que foi aquecido em chapa aquecedora (Biomixer DB-IVAC), permanecendo sob refluxo contínuo.

O solvente evapora e se condensa sobre o material sólido. Neste processo, os solutos são concentrados no balão. Após a destilação, os produtos foram direcionados para um rotaevaporador (Fisatom 803), acoplado a uma bomba de vácuo (New Pump) para evaporação do solvente a temperatura abaixo do seu ponto de ebulição. O solvente hexano foi utilizado com uma relação de 1:20 e o tempo de extração foi de 5 horas.

\section{Extração com $\mathrm{CO}_{2}$ supercrítico}

O aparato experimental, como mostrado na Figura 1, para a extração dos óleos bioativos das microalgas com dióxido de carbono supercrítico se encontra no Laboratório de Termodinâmica Aplicada e Biocombustíveis (DEQ/UFRRJ) e é composto por um extrator de aço inoxidável $316 \mathrm{~S}$ de $42 \mathrm{~mL}$ de volume, com telas de 260 mesh no topo e no fundo para evitar a passagem de qualquer material, evitando o entupimento da linha. $O$ extrator foi acoplado a um banho termostático (Tecnal) para controle da temperatura durante o processo de extração. Uma bomba de alta pressão (Palm modelo G100), específica para bombeamento de $\mathrm{CO}_{2}$, foi responsável pela alimentação do solvente. A vazão de $\mathrm{CO}_{2}$ foi de, aproximadamente, de $6,04 \mathrm{~mL} / \mathrm{min}$. 
Figura 1 - Esquema da unidade supercrítica inserida no Laboratório de

Termodinâmica Aplicada e Biocombustíveis, sendo A: Cilindro de $\mathrm{CO}_{2}$; B: Bomba de alta pressão; C: Banho térmico; D: Extrator; E: Válvula micrométrica; F: Amostra; G: Medidor de vazão (Adaptado de Mendes, 2002).

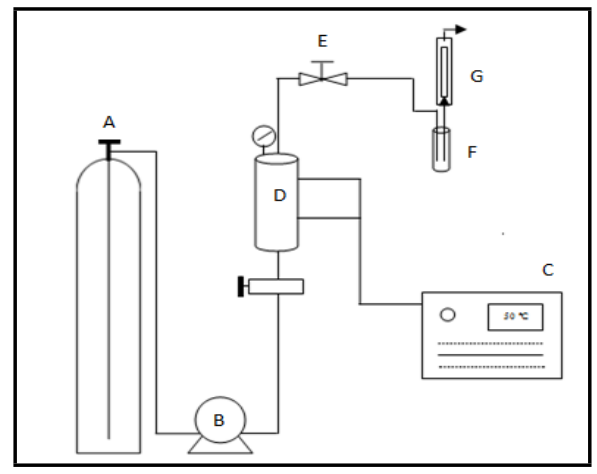

O extrator foi alimentado com cerca de $10 \mathrm{~g}$ da biomassa microalgal e, após sua alimentação, foi adicionado à linha da bomba de alta pressão. Ao extrator foi acoplado o banho térmico para circulação da água a fim de atingir e manter a temperatura desejada.

Os ensaios foram realizados seguindo o planejamento de experimentos com o tempo máximo de extração de 440 minutos, onde foi observada a saturação da curva de extração. Foi utilizada a técnica de despressurização através de uma válvula micrométrica que controla o fluxo e recolhe as amostras em tempos determinados. A amostragem variou de acordo com a condição experimental, visto que a extração pode ser mais lenta ou mais rápida. Para todas as condições de 100, 160 bar e 500 bar, a mesma foi realizada a cada 20 minutos até o esgotamento da extração. Para pressões de 300 - 440 bar, observou-se a presença de extrato já em 10 minutos e a amostragem, então, foi realizada considerando esse intervalo. Com a redução da pressão, a amostra é recuperada em um tubo de polipropileno, previamente pesado. Após cada coleta, o tubo foi mantido imerso em um banho com gelo para que não houvesse degradação do extrato.

\section{MODELO MATEMÁTICO}

A teoria do modelo de Esquível et al. (1999) se trata do uso da equação cinética de crescimento microbiano proposto por Monod para representar o rendimento da extração em função do tempo de extração. Esquível et al. (1999) propuseram este modelo para descrever curvas de extração globais e por ser um modelo empírico, não considera interações entre o soluto e a matriz sólida e tem apenas dois parâmetros de ajuste. Conforme apresentado na Equação 1.

$$
e=e_{\lim i\left(\frac{t}{b+t}\right)}
$$

Sendo e a razão entre a massa de óleo recuperada em função do tempo (t) e a massa inicial (M) de carga livre de soluto; $\mathbf{e}_{\text {lim }}$ o parâmetro ajustável, que representa o valor de "e" para um tempo infinito de extração e b parâmetro ajustável dependente da vazão mássica, temperatura e pressão, e não possui significado físico. $\mathrm{O}$ parâmetro $\mathrm{e}_{\lim }$ pode presumidamente depender apenas do material a ser extraído, desde que este seja igual ao conteúdo inicial de óleo do 
mesmo, e, ao se plotar a curva de rendimento (e) pelo tempo, a divisão " $\mathrm{e}_{\lim } / \mathrm{b}$ " representa a inclinação inicial da curva de extração.

O desvio relativo médio (DRM \%) foi calculado através da Equação 2, sendo $\mathrm{N}_{\exp } \mathrm{o}$ número de ponto experimentais utilizados.

$$
D R M \%=\frac{100}{N_{\text {exp }}} \sum\left|\left(\frac{(\text { e experimental })-(\text { e calculado })}{\text { e experimental }}\right)\right|
$$

\section{Resultados e Discussão}

O rendimento experimental (e \%) foi calculado de acordo com a Equação 3.

$$
e \%=\frac{\text { massa extraída }}{\text { massa de cargalivre de soluto }}
$$

(3)

sendo massa extraída, a massa de óleo extraído e massa de carga livre de soluto, a massa da microalga sem a presença do óleo.

Para o cálculo da massa de carga livre de soluto, é necessária a porcentagem padrão de óleo extraído no método convencional. A partir da realização deste experimento, obteve-se o valor de $16,73 \%$ de óleo extraído, através da extração usando soxhlet e hexano como solvente. Pode-se observar que, quanto maior a pressão, maior o rendimento em óleo, pois em geral, um aumento na pressão, a temperatura constante, causa um aumento na densidade do fluido supercrítico, elevando assim o seu poder solvente.

Já o efeito da temperatura na densidade é mais complexo; pode-se afirmar que, a pressão constante, um aumento na temperatura leva a uma diminuição na densidade do solvente supercrítico. Mudanças na temperatura, além de influenciarem na densidade do solvente, também influenciam na pressão de vapor e na energia cinética das moléculas de soluto. Em temperaturas próximas do ponto crítico, o efeito da diminuição da densidade do solvente supercrítico é mais pronunciado (Pereira \& Meireles, 2010).

A pressão de vapor do soluto aumenta com a temperatura e tende a aumentar a solubilidade, enquanto que a temperatura tem um efeito contrário sobre a densidade do solvente e a solubilidade. Isto pode ser observado nas condições de 300 bar $-100{ }^{\circ} \mathrm{C}$ e 440 bar $-90{ }^{\circ} \mathrm{C}$ e $500 \mathrm{bar}-70{ }^{\circ} \mathrm{C}$, onde quanto maior a pressão, maior o rendimento em óleo. Em contrapartida nas maiores temperaturas, isso se deve ao efeito de pressão de vapor e melhor foram os rendimentos em óleo.

O modelo de Esquível et al. (1999), aplicado a esse trabalho, consegue representar toda a curva de extração, tanto a parte rápida, quanto a parte mais lenta da curva. Os desvios foram baixos e variaram de $2,1742 \%$ a $14,3327 \%$. Os valores dos rendimentos experimentais e calculados e dos respectivos desvios podem ser vistos na Tabela 1 e as curvas de extração na Figura 2, para as diferentes condições operacionais. 


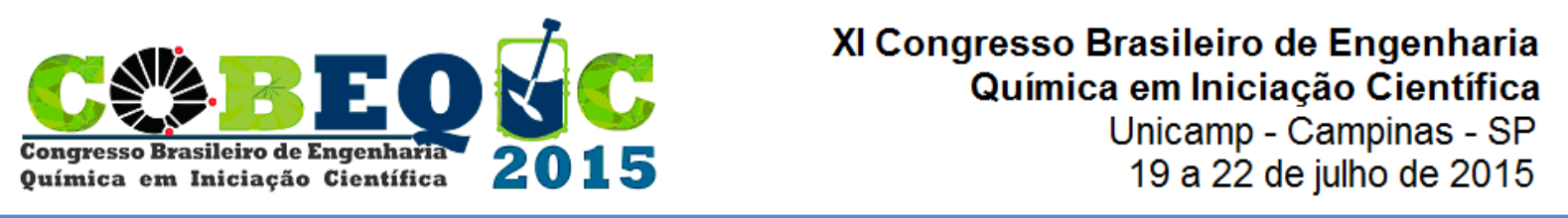

Tabela 1 - Rendimento calculado, os parâmetros e desvio relativo obtido para o modelo de Esquível et al. (1999) nas diferentes condições de pressão e temperatura.

\begin{tabular}{cccccccc}
\hline Ensaios & $\begin{array}{c}\text { Pressão } \\
(\mathbf{b a r})\end{array}$ & $\begin{array}{c}\text { Temperatura } \\
(\mathbf{K})\end{array}$ & $\mathbf{e}_{\text {Experimental }}$ & $\mathbf{e}_{\text {Calculado }}$ & $\mathbf{e}_{\text {lim }}$ & $\mathbf{b}$ & DR (\%) \\
\hline 1 & 160 & 323 & 0,0372 & 0,0365 & 0,1095 & 159,9391 & 3,6976 \\
2 & 160 & 363 & 0,0336 & 0,0318 & 0,0358 & 12,5926 & 4,5514 \\
3 & 440 & 323 & 0,4143 & 0,3655 & 0,8598 & 283,9498 & 7,0275 \\
4 & 440 & 363 & 2,5866 & 2,6359 & 4,8185 & 206,9947 & 6,0219 \\
5 & 300 & 313 & 0,1957 & 0,1740 & 0,3023 & 110,5831 & 14,0743 \\
6 & 300 & 373 & 3,3427 & 3,4524 & 69,3559 & 5089,2980 & 2,1742 \\
7 & 100 & 343 & 0,0600 & 0,0644 & 0,1721 & 233,9638 & 4,1439 \\
8 & 500 & 343 & 3,1038 & 3,2176 & 16,2384 & 1699,6610 & 5,5364 \\
9 & 300 & 343 & 0,1570 & 0,1615 & 0,4635 & 224,3296 & 3,3272 \\
\hline
\end{tabular}

Pereira (2009) relata que ao contrário do que foi observado por Esquível et al. (1999), o parâmetro $e_{\text {lim }}$ também sofreu variações com a temperatura e a pressão. A comprovação dessa

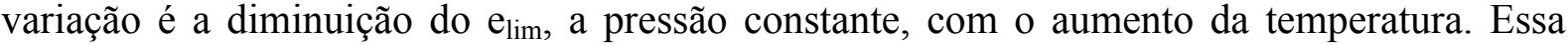
dependência também foi observada em Silva et al. (2008).

Figura 3 - As curvas de extração para todas as pressões e temperaturas investigadas, sendo (a) Nas condições de 100 bar $-70{ }^{\circ} \mathrm{C}, 160$ bar $-50{ }^{\circ} \mathrm{C}$ e 160 bar- $90^{\circ} \mathrm{C}$.; (b) Nas condições de 300 bar $-100{ }^{\circ} \mathrm{C}, 440$ bar $-90^{\circ} \mathrm{C}$ e 500 bar- $70^{\circ} \mathrm{C}$; (c) Nas condições de 300 bar $-40{ }^{\circ} \mathrm{C}, 300$ bar $-70{ }^{\circ} \mathrm{C}$ (ponto central) e 440 bar- $50^{\circ} \mathrm{C}$.
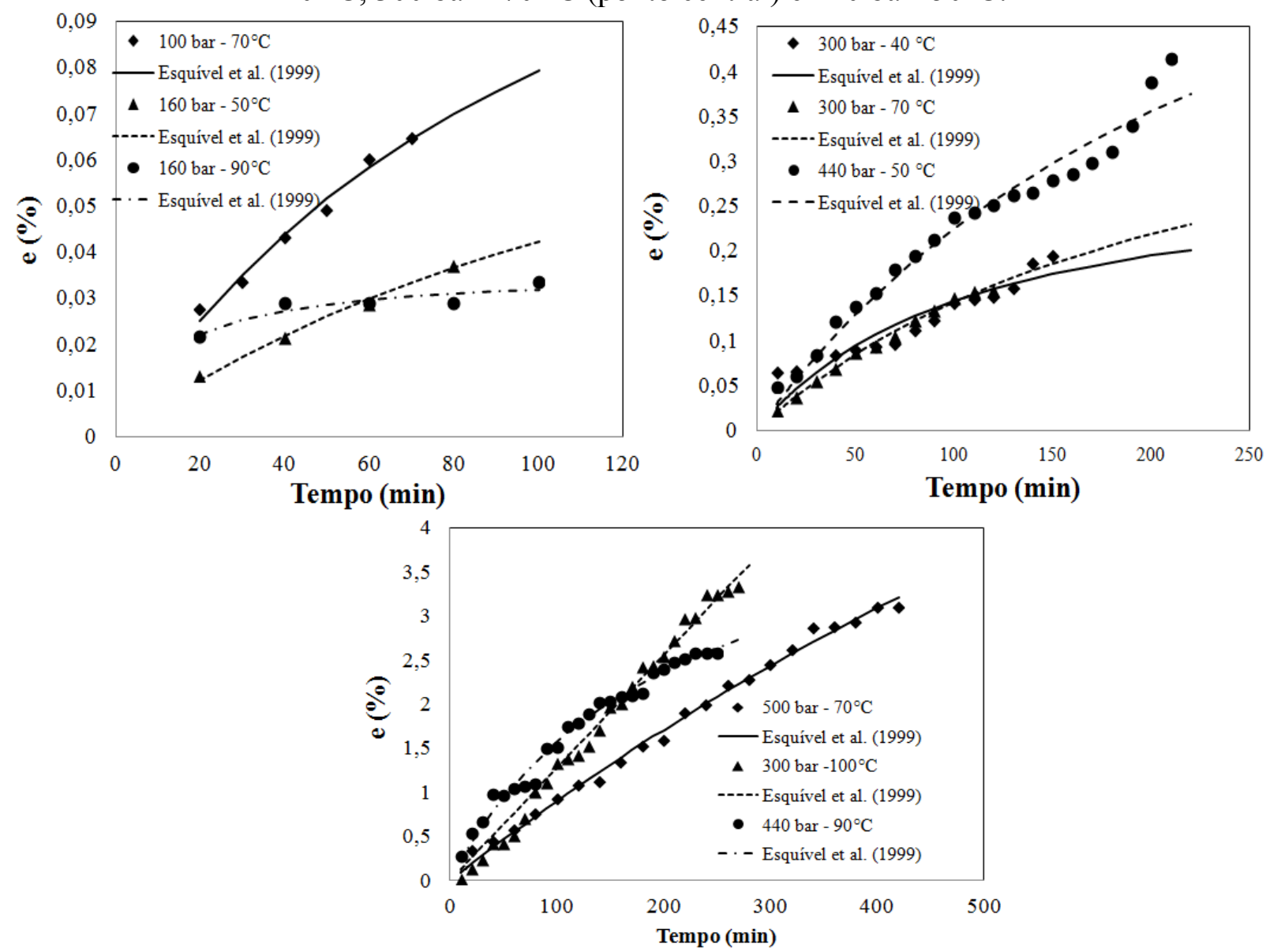
Nos trabalhos que estudaram o modelo de Esquível et al. (1999) com extração de compostos usando fluido supercrítico, como o de Pereira (2009) e o reportado por Silva et al. (2008), os valores de $\mathrm{e}_{\mathrm{lim}}$ foram baixos, para algumas condições operacionais. No caso do $\mathrm{e}_{\mathrm{lim}}$ deste trabalho, também foram baixos com exceção da condição de 300 bar e $100{ }^{\circ} \mathrm{C}$.

\section{CONCLUSÃO}

Inicialmente, pode-se concluir que a extração supercrítica foi uma técnica promissora para a extração do óleo da microalga Desmodesmus sp., usando o $\mathrm{CO}_{2}$ supercrítico. Os melhores resultados obtidos foram nas condições de $300 \mathrm{bar}-100{ }^{\circ} \mathrm{C}, 500$ bar $-70{ }^{\circ} \mathrm{C}$ e 440 bar e $90{ }^{\circ} \mathrm{C}$, alcançando valores de 3,342\%, 3,103\% e 2,586\%, respectivamente. O modelo empírico estudado, Esquível et al. (1999) é apto a representar a extração do óleo da microalga em questão.

\section{REFERÊNCIAS}

BRENNAN, L., OWEDE, P. Biofuels from microalgae- A rewiew of technologies for production, processing, and extractions of biofuels and co- products. Rewable and Sustainable Rewiews. 14, 557-577, 2010.

ESQUÍVEL, M. M.; BERNARDO-GIL, M. G.; KING, M. B. Mathematical models for supercritical extraction of olive husk oil. Journal of Supercritical Fluids. v.16, p. 4358,1999 .

MENDES, M.; PESSOA, F.; ULLER, A. An economic evaluation based on an experimental study of the vitamin E concentration present in deodorizer distillate of soybean oil using supercritical CO2. The Journal of Supercritical Fluids, v. 23, p. 257-265, 2002.

PEREIRA, C.G.; MEIRELES, M.A.A. Supercritical fluid extraction of bioactive compounds: fundamentals, applications and economic perspectives. Food and Bioprocess Technology,v. 3, p. 340-372, 2010.

PEREIRA, C. S. S. Avaliação De Diferentes Tecnologias Na Extração Do Óleo Do Pinhãomanso (Jatropha curcas L), dissertação de mestrado, UFRRJ, 2009.

SILVA, C. F.; MENDES, M. F.; PESSOA, F. L. P.; QUEIROZ, E. M. Supercritical carbon dioxide extraction of macadamia (Macadamia integrifolia) nut oil: experiments and modeling. Braz. J. Chem. Eng..vol.25 no.1, 2008.

SKULBERG, O. In: RICHMOND, A. Handbook of microalgal culture: biotechnology and applied phycology. Oxford: Blackwell Science, 2004. 